International Journal of Pure and Applied Mathematics

Volume 90 No. 4 2014, 485-500

ISSN: 1311-8080 (printed version); ISSN: 1314-3395 (on-line version)

url: http://www.ijpam.eu

doi: http://dx.doi.org/10.12732/ijpam.v90i4.10

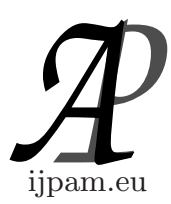

\title{
ON THE EXTENSION OF ARMENDARIZ RINGS RELATIVE TO A MONOID
}

\author{
Ayoub Elshokry ${ }^{1}$, Eltiyeb $\mathrm{Ali}^{2}$, Liu Zhong-Kui ${ }^{3}$ \\ $1,2,3$ Department of Mathematics \\ Northwest Normal University \\ Lanzhou, 730070, P.R. CHINA \\ ${ }^{1,2}$ Department of Mathematics \\ Khartoum University \\ Omdurman, SUDAN
}

\begin{abstract}
For a monoid $M$, we introduce the concept of 3-M-Armendariz rings, which is a generalization of $M$-Armendariz rings, and investigate its properties. The results prove that the subrings of $3-M$-Armendariz rings are $3-M$-Armendariz rings. Every ring satisfying condition $(P)$ is $3-M$-Armendariz for any unique product monoid $M$. If a ring $R$ is 3 - $M$-Armendariz and satisfies condition $(P)$, then $S_{3}(R)$ is $3-M$-Armendariz. Sufficient and necessary conditions are given for a ring $R$ to be 3 - $M$-Armendariz.
\end{abstract}

AMS Subject Classification: 16U20, 16N60, 16U99

Key Words: Armendariz ring, 3-Armendariz ring, 3- $M$-Armendariz ring

\section{Introduction}

Throughout this paper, $R$ and $M$ denote an associative ring, not necessary with identity and a monoid, respectively. Given a ring $R$, the polynomial ring over $R$ is denoted by $R[x]$. The study of Armendariz rings was initiated by Armendariz [5] and Rege and Chhawchharia [8]. A ring $R$ is called Armendariz if whenever

Received: October 30, 2013

(C) 2014 Academic Publications, Ltd.

$\S$ Correspondence author url: www.acadpubl.eu 
polynomials $f(x)=a_{0}+a_{1} x+\cdots+a_{n} x^{n}, g(x)=b_{0}+b_{1} x+\cdots+b_{m} x^{m} \in R[x]$ satisfy $f(x) g(x)=0$, then $a_{i} b_{j}=0$, for all $0 \leq i \leq n, 0 \leq j \leq m$. (The converse is always true.) Some properties of Armendariz rings have been studied in Rege and Chhawchharia [8], Anderson and Camillo [3], Kim and Lee [9], Huh et al. [1], and Lee and Wong [11]. Suiyi [14] introduced the notion of 3-Armendariz rings. A ring $R$ is called a 3-Armendariz ring if whenever polynomials $f(x)=$ $a_{0}+a_{1} x+\cdots+a_{n} x^{n}, g(x)=b_{0}+b_{1} x+\cdots+b_{m} x^{m}, h(x)=c_{0}+c_{1} x+\cdots+c_{r} x^{r} \in$ $R[x]$, satisfy $f(x) g(x) h(x)=0$, then $a_{i} b_{j} c_{k}=0$, for all $0 \leq i \leq n, 0 \leq j \leq$ $m, 0 \leq k \leq r$. Zhongkui [7], studied a generalization of Armendariz rings, which are called $M$-Armendariz rings, where $M$ is a monoid. A ring $R$ is called $M$ Armendariz if whenever elements $\alpha=a_{1} g_{1}+\cdots+a_{n} g_{n}, \beta=b_{1} h_{1}+\cdots+b_{m} h_{m} \in$ $R[M]$, satisfy $\alpha \beta=0$, then $a_{i} b_{j}=0$ for each $i, j$, where $g_{i}, h_{j} \in M$. A ring $R$ is called reduced if it has no nonzero nilpotent elements. Reduced rings are Armendariz by Armendariz [5, lemma 1.1] and subrings of a Armendariz ring are also Armendariz. A ring is called abelian if every idempotent is central. Armendariz ring are abelian by Kim and Lee [9]. Subrings of a $M$-Armendariz ring are also $M$-Armendariz by Zhongkui [7]. Subrings of a 3-Armendariz ring are also 3-Armendariz by Suiyi [14].

Recall that a monoid $M$ is called a u.p.-monoid (unique product monoid) if for any two non-empty finite subsets $A, B \subseteq M$ there exists an element $g \in M$ uniquely presented in the form $a b$ where $a \in A$ and $b \in B$. The class of u.p.-monoids is quite large and important (see Birkenmeier and Park [6], Passman [4]). For example, this class includes the right or left ordered monoids, submonoids of a free group, and torsion-free nilpotent groups. Every u.p.monoid $M$ has non unity element of finite order.

Motivated by results in Suiyi [14], Zhongkui [7], Rege and Chhawchharia [8] and Kim and Lee [9], we will investigate a generalization of $M$-Armendariz rings, which we call 3 - $M$-Armendariz rings.

In the following, e will always stand for the identity of $M$.

\section{3-M-Armendariz Rings}

Definition 2.1. Let $M$ be a monoid. A ring $R$ is called a $3-M$-Armendariz ring if whenever elements $\alpha=a_{1} g_{1}+\cdots+a_{n} g_{n}, \beta=b_{1} h_{1}+\cdots+b_{m} h_{m}$ and $\gamma=c_{1} l_{1}+\cdots+c_{r} l_{r} \in R[M]$, satisfy $\alpha \beta \gamma=0$, then $a_{i} b_{j} c_{k}=0$ for each $i, j$ and $k$, where $a_{i}, b_{j}, c_{k} \in R$ and $g_{i}, h_{j}, l_{k} \in M$.

Every $M$-Armendariz ring is 3 - $M$-Armendariz, but the converse is not true 
by the following example.

Example 2.2. Let $M$ a monoid with $|M| \geq 2$. Then $B_{6}^{e}(\mathbb{Z})$ is $3-M$ Armendariz, but not $M$-Armendariz, where the ring $B_{6}^{e}(\mathbb{Z})$ is defined as following see([12]):

$$
B_{n=6}^{e}(\mathbb{Z})=\sum_{i=1}^{k+1} \sum_{j=k+i-1}^{6} \mathbb{Z} E_{i, j} .
$$

Proof. By Suiyi [14, Example 1], we have $\left(B_{6}^{e}(\mathbb{Z})\right)^{3}=0$. So $B_{6}^{e}(\mathbb{Z})$ is a 3 $M$-Armendariz ring. But $B_{6}^{e}(\mathbb{Z})$ is not $M$-Armendariz, because for any $g \neq e$, $\left(E_{13} e+\left(E_{13}-E_{14}\right) g\right)\left(E_{46} e+\left(E_{36}+E_{46}\right) g\right)=0$ in $B_{6}^{e}(\mathbb{Z})[M]$, but $E_{13} \cdot\left(E_{36}+\right.$ $\left.E_{46}\right)=E_{16} \neq 0$.

Proposition 2.3. Every subring of 3-M-Armendariz rings is 3-M-Armendariz.

Proof. It is obvious.

We introduce the following notation (see [14]).

Condition (P): For all $a, b, c \in R$, if $(a b c)^{2}=0$, then $a b c=0$.

Lemma 2.4. [13, Proposition 1]. If $R$ is a reduced ring, then $R$ satisfies the condition $(P)$, but the converse is not true.

Lemma 2.5. [6, Lemma 1.1] Assume $M$ is a u.p.-monoid. Then $M$ is cancellative (i.e., for $g, h, x \in M$, if $g x=h x$ or $x g=x h$, then $g=h$ ).

Theorem 2.6. Let $M$ be a u.p.-monoid and $R$ a ring satisfying condition $(P)$. Then $R$ is $3-M$-Armendariz.

Proof. Let $\alpha=a_{1} g_{1}+\cdots+a_{n} g_{n}, \beta=b_{1} h_{1}+\cdots+b_{m} h_{m}$ and $\gamma=c_{1} l_{1}+$ $\cdots+c_{r} l_{r} \in R[M]$, be such that $\alpha \beta \gamma=0$. We claim $a_{i} b_{j} c_{k}=0$ for each $i, j, k$. We proceed by induction on $n, m$ and $r$. Let $n=1$. Then $\alpha=a_{1} g_{1}$. So

$$
\left(a_{1} g_{1}\right)\left(b_{1} h_{1}+\cdots+b_{m} h_{m}\right)\left(c_{1} l_{1}+\cdots+c_{r} l_{r}\right)=0 .
$$

If $m=1$, then $a_{1} b_{1} c_{1} g_{1} h_{1} l_{1}+\cdots+a_{1} b_{1} c_{r} g_{1} h_{1} l_{r}=0$. By Lemma $2.5, g_{1} h_{1} l_{i} \neq$ $g_{1} h_{1} l_{j}$ for $i \neq j$. Thus, $a_{1} b_{1} c_{k}=0$ for all $k$. The case $r=1$, is proved by similar argument. Now suppose that $m>1$ and $r>1$. Since $M$ is a u.p.-monoid, there exist $j, k$ with $1 \leq j \leq m$ and $1 \leq k \leq r$ such that $h_{j} l_{k}$ is uniquely presented by considering two subsets $A=\left\{h_{1}, \cdots, h_{m}\right\}$ and $B=\left\{l_{1}, \cdots, l_{r}\right\}$. Without loss of generality, we may assume that $j=1, k=1$. Thus, by Lemma 2.5 it follows 
that $g_{1} h_{1} l_{1} \neq g_{1} h_{j} l_{k}$ for any $j \neq 1$ or $k \neq 1$. Hence we have $a_{1} b_{1} c_{1}=0$. Since $R$ satisfies condition $(P), c_{1} a_{1} b_{1}=0$ follows. Now from $(*)$, it follows that

$$
\left(c_{1} a_{1} g_{1}\right)\left(b_{1} h_{1}+\cdots+b_{m} h_{m}\right)\left(c_{1} l_{1}+\cdots+c_{r} l_{r}\right)=0 .
$$

Thus,

$$
\left(c_{1} a_{1} g_{1}\right)\left(b_{2} h_{2}+\cdots+b_{m} h_{m}\right)\left(c_{1} l_{1}+\cdots+c_{r} l_{r}\right)=0 .
$$

By induction, we have $c_{1} a_{1} b_{j} c_{k}=0$ for all $j$ and $k$. Then $c_{1} a_{1} b_{j} c_{1}=0$ for all $j$. Thus, $\left(c_{1} a_{1} b_{j}\right)^{2}=0$, which implies that $c_{1} a_{1} b_{j}=0$ for all $j$ since $R$ satisfies condition $(P)$. So, again by condition $(P)$, we have $a_{1} b_{j} c_{1}=0$ for all $j$. Thus, by $(*)$ it follows that

$$
\left(a_{1} g_{1}\right)\left(b_{1} h_{1}+\cdots+b_{m} h_{m}\right)\left(c_{2} l_{2}+\cdots+c_{r} l_{r}\right)=0
$$

By induction, we have $a_{1} b_{j} c_{k}=0$ for all $j$ and $k$ with $1 \leq j \leq m, 2 \leq k \leq r$. Thus $a_{1} b_{j} c_{k}=0$ for all $j$ and $k$. If $m=1$ or $r=1$, then by analogy with the above argument, the result follows. Now suppose that $n>1$. Consider two subsets $A=\left\{g_{1}, \cdots, g_{n}\right\}$ and $B=\left\{h_{j} l_{k} \mid 1 \leq j \leq m, 1 \leq k \leq r\right\}$. Since $M$ is a u.p.-monoid, there exist $i, j, k$ with $1 \leq i \leq n, 1 \leq j \leq m, 1 \leq k \leq r$, such that $g_{i} h_{j} l_{k}$ is uniquely presented. Without loss of generality, we may assume that $i=1, j=1, k=1$. Thus, $a_{1} b_{1} c_{1}=0$, which implies $b_{1} c_{1} a_{1}=0$. From $\alpha \beta \gamma=0$ it follows

$$
b_{1} c_{1}\left(a_{2} g_{2}+\cdots+a_{n} g_{n}\right)\left(b_{1} h_{1}+\cdots+b_{m} h_{m}\right)\left(c_{1} l_{1}+\cdots+c_{r} l_{r}\right)=0 .
$$

By induction, we have $b_{1} c_{1} a_{i} b_{j} c_{k}=0$, for all $i, j, k$. Particularly, $b_{1} c_{1} a_{i} b_{1} c_{1}=0$, which implies that $a_{i} b_{1} c_{1}=0$ for all $i$. Thus, by condition $(\mathrm{P})$, we have $c_{1} a_{i} b_{1}=0$ for all $i$. From $\alpha \beta \gamma=0$, it follows that

$$
\begin{aligned}
0= & c_{1}\left(a_{1} g_{1}+\cdots+a_{n} g_{n}\right)\left(b_{1} h_{1}+\cdots+b_{m} h_{m}\right)\left(c_{1} l_{1}+\cdots+c_{r} l_{r}\right) \\
= & c_{1}\left(a_{1} g_{1}+\cdots+a_{n} g_{n}\right)\left(b_{2} h_{2}+\cdots+b_{m} h_{m}\right)\left(c_{1} l_{1}+\cdots+c_{r} l_{r}\right) \\
& +c_{1}\left(a_{1} g_{1}+a_{2} g_{2}+\cdots+a_{n} g_{n}\right)\left(b_{1} h_{1}\right)\left(c_{1} l_{1}+c_{2} l_{2}+\cdots+c_{r} l_{r}\right) \\
= & c_{1}\left(a_{1} g_{1}+\cdots+a_{n} g_{n}\right)\left(b_{2} h_{2}+\cdots+b_{m} h_{m}\right)\left(c_{1} l_{1}+\cdots+c_{r} l_{r}\right) .
\end{aligned}
$$

By induction, we have $c_{1} a_{i} b_{j} c_{k}=0$ for all $i, j, k$. Particularly $c_{1} a_{i} b_{j} c_{1}=0$ for all $i, j$. Since $R$ satisfies condition $(P)$, it follows that $a_{i} b_{j} c_{1}=0$ for all $i$ and $j$. Hence we have

$$
\left(a_{1} g_{1}+\cdots+a_{n} g_{n}\right)\left(b_{1} h_{1}+\cdots+b_{m} h_{m}\right)\left(c_{2} l_{2}+\cdots+c_{r} l_{r}\right)=0 .
$$

By induction, it follows that $a_{i} b_{j} c_{k}=0$, for all $i, j$, and $k$. 
Let $(M, \leq)$ be an ordered monoid. If for any $g, g^{\prime}, h \in M, g<g^{\prime}$ implies that $g h<g^{\prime} h$ and $h g<h g^{\prime}$, then $(M, \leq)$ is called a strictly ordered monoid.

Corollary 2.7. Let $M$ be a strictly totally ordered monoid and $R$ a ring satisfying condition $(P)$. Then $R$ is $3-M$-Armendariz.

Corollary 2.8. If a ring $R$ satisfies condition $(P)$, then $R$ is $3-\mathbb{Z}$-Armendariz, that is, for any $\alpha=a_{-m} x^{-m}+a_{-(m-1)} x^{-(m-1)}+\cdots+a_{p} x^{p}, \beta=b_{-n} x^{-n}+$ $b_{-(n-1)} x^{-(n-1)}+\cdots+b_{q} x^{q}$ and $\gamma=c_{-t} x^{-t}+c_{-(t-1)} x^{-(t-1)}+\cdots+c_{s} x^{s} \in$ $R\left[x, x^{-1}\right]$, if $\alpha \beta \gamma=0$, then $a_{i} b_{j} c_{k}=0$ for $-m \leq i \leq p,-n \leq j \leq q$ and $-t \leq k \leq s$.

It was shown in Zhongkui [7], Proposition 1.4, that if $I$ is a reduced ideal of $R$ such that $R / I$ is $M$-Armendariz, then $R$ is $M$-Armendariz. Here we have the following result for $3-M$-Armendariz property.

Theorem 2.9. Let $M$ be a strictly totally ordered monoid and $I$ an ideal of $R$. If $I$ is reduced and $R / I$ is 3-M-Armendariz, then $R$ is 3-M-Armendariz.

Proof. Let $\alpha, \beta, \gamma \in R[M]$ be such that $\alpha \beta \gamma=0$. We write $\alpha=a_{1} g_{1}+\cdots+$ $a_{n} g_{n}, \beta=b_{1} h_{1}+\cdots+b_{m} h_{m}$ and $\gamma=c_{1} l_{1}+\cdots+c_{r} l_{r} \in R[M]$, with

$$
g_{1}<g_{2}<\cdots<g_{n}, h_{1}<h_{2}<\cdots<h_{m}, l_{1}<l_{2}<\cdots<l_{r} .
$$

We will use transfinite induction on the strictly totally ordered set $(M, \leq)$ to show that $a_{i} b_{j} c_{k}=0$, for any $i, j$ and $k$. Note that in $(R / I)[M],\left(\bar{a}_{1} g_{1}+\bar{a}_{2} g_{2}+\right.$ $\left.\cdots+\bar{a}_{n} g_{n}\right)\left(\bar{b}_{1} h_{1}+\bar{b}_{2} h_{2}+\cdots+\bar{b}_{m} h_{m}\right)\left(\bar{c}_{1} l_{1}+\bar{c}_{2} l_{2}+\cdots+\bar{c}_{r} l_{r}\right)=0$. Thus, we have $a_{i} b_{j} c_{k} \in I$, for all $i, j$ and $k$, with $1 \leq i \leq n, 1 \leq j \leq m$ and $1 \leq k \leq r$, since $R / I$ is 3 - $M$-Armendariz. If there exist $1 \leq i \leq n, 1 \leq j \leq m$ and $1 \leq k \leq r$, such that $g_{i} h_{j} l_{k}=g_{1} h_{1} l_{1}$, then $g_{1} \leq g_{i}, h_{1} \leq h_{j}$ and $l_{1} \leq l_{k}$. If $g_{1}<g_{i}$, then $g_{1} h_{1} l_{1}<g_{i} h_{1} l_{1} \leq g_{i} h_{j} l_{k}=g_{1} h_{1} l_{1}$, a contradiction, thus $g_{1}=g_{i}$. Similarly, $h_{1}=h_{j}$ and $l_{1}=l_{k}$. Hence $a_{1} b_{1} c_{1}=0$. Now suppose that $w \in M$ is such that for any $g_{i}, h_{j}$ and $l_{k}$, if $g_{i} h_{j} l_{k}<w$, then $a_{i} b_{j} c_{k}=0$. We will show that $a_{i} b_{j} c_{k}=0$, for any $g_{i}, h_{j}$ and $l_{k}$, with $g_{i} h_{j} l_{k}=w$. Set $X=\left\{\left(g_{i}, h_{j}, l_{k}\right) \mid g_{i} h_{j} l_{k}=w\right\}$. Then $X$ is a finite set. We write $X$ as $\left\{\left(g_{i_{t}}, h_{j_{t}}, l_{k_{t}}\right) \mid t=1,2, \cdots, u\right\}$ such that

$$
g_{i_{1}}<g_{i_{2}}<\cdots<g_{i_{u}} \text {. }
$$

We claim that

$$
h_{j_{u}} l_{k_{u}}<\cdots<h_{j_{2}} l_{k_{2}}<h_{j_{1}} l_{k_{1}} .
$$

In fact, if $h_{j_{1}} l_{k_{1}}<h_{j_{2}} l_{k_{2}}$, then

$$
w=g_{i_{1}} h_{j_{1}} l_{k_{1}}<g_{i_{1}} h_{j_{2}} l_{k_{2}}<g_{i_{2}} h_{j_{2}} l_{k_{2}}=w
$$


a contradiction. If $h_{j_{1}} l_{k_{1}}=h_{j_{2}} l_{k_{2}}$, then from $g_{i_{1}} h_{j_{1}} l_{k_{1}}=w=g_{i_{2}} h_{j_{2}} l_{k_{2}}$ it follows that $g_{i_{1}}=g_{i_{2}}$, a contradiction again. Thus, $h_{j_{2}} l_{k_{2}}<h_{j_{1}} l_{k_{1}}$. Similarly we have the claim. For any $t \geq 2, g_{i_{1}} h_{j_{t}} l_{k_{t}}<g_{i_{t}} h_{j_{t}} l_{k_{t}}=w$, and thus, by induction hypothesis, we have $a_{i_{1}} b_{j_{t}} c_{k_{t}}=0$. Since $b_{j_{t}} c_{k_{t}} I a_{i_{1}} \subseteq I,\left(b_{j_{t}} c_{k_{t}} I a_{i_{1}}\right)^{2}=0$, and $I$ is reduced, we have $b_{j_{t}} c_{k_{t}} I a_{i_{1}}=0$ for any $t \geq 2$. Thus, for any $t \geq 2$,

$$
\left(a_{i_{t}} b_{j_{t}} c_{k_{t}}\right)\left(a_{i_{1}} b_{j_{1}} c_{k_{1}}\right)^{2} \subseteq\left(a_{i_{t}} b_{j_{t}} c_{k_{t}}\right) I\left(a_{i_{1}} b_{j_{1}} c_{k_{1}}\right)=a_{i_{t}}\left(b_{j_{t}} c_{k_{t}} I a_{i_{1}}\right) b_{j_{1}} c_{k_{1}}=0 .
$$

Now, from

$$
\sum_{\left(g_{i}, h_{j}, l_{k}\right) \in X}\left(a_{i} b_{j} c_{k}\right)=\sum_{t=1}^{u} a_{i t} b_{j_{t}} c_{k_{t}}=0
$$

it follows that

$$
\left(\sum_{t=1}^{u} a_{i_{t}} b_{j_{t}} c_{k_{t}}\right)\left(a_{i_{1}} b_{j_{1}} c_{k_{1}}\right)^{2}=\left(a_{i_{1}} b_{j_{1}} c_{k_{1}}\right)^{3}=0 .
$$

Since $a_{i_{1}} b_{j 1} c_{k_{1}} \in I$ and $I$ is reduced, we have $a_{i_{1}} b_{j_{1}} c_{k_{1}}=0$. Thus, $\sum_{t=2}^{u} a_{i_{t}} b_{j_{t}} c_{k_{t}}=$ 0 . Multiplying $\left(a_{i_{2}} b_{j_{2}} c_{k_{2}}\right)^{2}$ on $\sum_{t=2}^{u} a_{i_{t}} b_{j_{t}} c_{k_{t}}=0$, from the right-hand side, we obtain $a_{i_{2}} b_{j_{2}} c_{k_{2}}=0$, by the same way as the above. Continuing this process, we can prove $a_{i_{t}} b_{j_{t}} c_{k_{t}}=0$, for $t=1,2, \cdots, u$. Thus, $a_{i} b_{j} c_{k}=0$ for any $i, j$ and $k$ where $g_{i} h_{j} l_{k}=w$. Now the result follows.

Recall that a monoid $M$ is called torsion-free if the following property holds: if $g, h \in M$ and $k \geq 1$ are such that $g^{k}=h^{k}$, then $g=h$.

Corollary 2.10. Let $M$ be a commutative, cancellative, and torsion-free monoid. If one of the following conditions holds, then $R$ is $3-M$-Armendariz.

1. $R$ satisfies condition $(P)$.

2. $R / I$ is $3-M$-Armendariz for some ideal $I$ of $R$, and $I$ is reduced.

Proof. If $M$ is commutative, cancellative, and torsion-free, then, by Ribenboim [10], there exists a compatible strict total order $\leq$ on $M$. Now the results follow from Corollary 2.7 and Theorem 2.9.

Proposition 2.11. Suppose that $R$ is 3 - $M$-Armendariz, $n \geq 3$. If $\alpha_{1}, \alpha_{2}$, $\cdots, \alpha_{n} \in R[M]$ are such that, $\alpha_{1} \alpha_{2} \cdots \alpha_{n}=0$, then $a_{1} a_{2} \cdots a_{n}=0$, where $a_{i}$ is a coefficient of $\alpha_{i}$.

Proof. It follows easily from the definition. 
Let $R$ be a ring. Define a ring $S_{3}(R)$ as follows:

$$
S_{3}(R)=\left\{\left(\begin{array}{lll}
a & b & c \\
0 & a & d \\
0 & 0 & a
\end{array}\right) \mid a, b, c, d \in R\right\} .
$$

Theorem 2.12. Let $M$ be a monoid with $|M| \geq 2$, and $R$ be $3-M$ Armendariz and satisfies condition $(P)$. Then $S_{3}(R)$ is 3-M-Armendariz.

Proof. Let $R$ be 3 - $M$-Armendariz and satisfies condition $(P)$. We show that $S_{3}(R)$ is 3 - $M$-Armendariz. Now we complete the proof by adapting the proof of Kim and Lee [9, Proposition 2]. It is easy to see that there exists an isomorphism of rings $S(R)[M] \rightarrow S(R[M])$ define by:

$$
\sum_{i=1}^{n}\left(\begin{array}{ccc}
a_{i} & b_{i} & c_{i} \\
0 & a_{i} & d_{i} \\
0 & 0 & a_{i}
\end{array}\right)_{i} \rightarrow\left(\begin{array}{ccc}
\sum_{i=1}^{n} a_{i} g_{i} & \sum_{i=1}^{n} b_{i} g_{i} & \sum_{i=1}^{n} c_{i} g_{i} \\
0 & \sum_{i=1}^{n} a_{i} g_{i} & \sum_{i=1}^{n} d_{i} g_{i} \\
0 & 0 & \sum_{i=1}^{n} a_{i} g_{i}
\end{array}\right)^{\circ}
$$

Suppose that $\alpha=A_{1} g_{1}+A_{2} g_{2}+\cdots+A_{n} g_{n}, \beta=B_{1} h_{1}+B_{2} h_{2}+\cdots+B_{m} h_{m}$ and $\gamma=C_{1} l_{1}+C_{2} l_{2}+\cdots+C_{r} l_{r} \in S_{3}(R)[M]$ are such that $\alpha \beta \gamma=0$, where $A_{i}, B_{j}, C_{k} \in S_{3}(R)$. We claim $A_{i} B_{j} C_{k}=0$ for each $i, j$ and $k$. Assume that

$$
A_{i}=\left(\begin{array}{ccc}
a_{i} & b_{i} & c_{i} \\
0 & a_{i} & d_{i} \\
0 & 0 & a_{i}
\end{array}\right), B_{j}=\left(\begin{array}{ccc}
a_{j}^{\prime} & b_{j}^{\prime} & c_{j}^{\prime} \\
0 & a_{j}^{\prime} & d_{j}^{\prime} \\
0 & 0 & a_{j}^{\prime}
\end{array}\right), C_{k}=\left(\begin{array}{ccc}
a_{k}^{\prime \prime} & b_{k}^{\prime \prime} & c_{k}^{\prime \prime} \\
0 & a_{k}^{\prime \prime} & d_{k}^{\prime \prime} \\
0 & 0 & a_{k}^{\prime \prime}
\end{array}\right)
$$

Then we have

$$
\begin{aligned}
&\left(\begin{array}{cccc}
\sum_{i=1}^{n} a_{i} g_{i} & \sum_{i=1}^{n} b_{i} g_{i} & \sum_{i=1}^{n} c_{i} g_{i} \\
0 & \sum_{i=1}^{n} a_{i} g_{i} & \sum_{i=1}^{n} d_{i} g_{i} \\
0 & 0 & \sum_{i=1}^{n} a_{i} g_{i}
\end{array}\right) \\
& \times\left(\begin{array}{cccc}
\sum_{j=1}^{m} a_{j}^{\prime} h_{j} & \sum_{j=1}^{m} b_{j}^{\prime} h_{j} & \sum_{j=1}^{m} c_{j}^{\prime} h_{j} \\
0 & \sum_{j=1}^{m} a_{j}^{\prime} h_{j} & \sum_{j=1}^{m} d_{j}^{\prime} h_{j} \\
0 & 0 & \sum_{j=1}^{m} a_{j}^{\prime} h_{j}
\end{array}\right) \\
& \times\left(\begin{array}{ccc}
\sum_{k=1}^{r} a_{k}^{\prime \prime} l_{k} & \sum_{k=1}^{r} b_{k}^{\prime \prime} l_{k} & \sum_{k=1}^{r} c_{k}^{\prime \prime} l_{k} \\
0 & \sum_{k=1}^{r} a_{k}^{\prime \prime} l_{k} & \sum_{k=1}^{r} d_{k}^{\prime \prime} l_{k} \\
0 & 0 & \sum_{k=1}^{r} a_{k}^{\prime \prime} l_{k}
\end{array}\right)=0 .
\end{aligned}
$$

Thus,

$$
\left(\sum_{i=1}^{n} a_{i} g_{i}\right)\left(\sum_{j=1}^{m} a_{j}^{\prime} h_{j}\right)\left(\sum_{k=1}^{r} a_{k}^{\prime \prime} l_{k}\right)=0
$$




$$
\begin{aligned}
& \left(\sum_{i=1}^{n} a_{i} g_{i}\right)\left(\sum_{j=1}^{m} a_{j}^{\prime} h_{j}\right)\left(\sum_{k=1}^{r} b_{k}^{\prime \prime} l_{k}\right)+\left(\sum_{i=1}^{n} a_{i} g_{i}\right)\left(\sum_{j=1}^{m} b_{j}^{\prime} h_{j}\right)\left(\sum_{k=1}^{r} a_{k}^{\prime \prime} l_{k}\right) \\
& +\left(\sum_{i=1}^{n} b_{i} g_{i}\right)\left(\sum_{j=1}^{m} a_{j}^{\prime} h_{j}\right)\left(\sum_{k=1}^{r} a_{k}^{\prime \prime} l_{k}\right)=0, \\
& \left(\sum_{i=1}^{n} a_{i} g_{i}\right)\left(\sum_{j=1}^{m} a_{j}^{\prime} h_{j}\right)\left(\sum_{k=1}^{r} c_{k}^{\prime \prime} l_{k}\right)+\left(\sum_{i=1}^{n} a_{i} g_{i}\right)\left(\sum_{j=1}^{m} b_{j}^{\prime} h_{j}\right)\left(\sum_{k=1}^{r} d_{k}^{\prime \prime} l_{k}\right) \\
& +\left(\sum_{i=1}^{n} b_{i} g_{i}\right)\left(\sum_{j=1}^{m} a_{j}^{\prime} h_{j}\right)\left(\sum_{k=1}^{r} d_{k}^{\prime \prime} l_{k}\right)+\left(\sum_{i=1}^{n} a_{i} g_{i}\right)\left(\sum_{j=1}^{m} c_{j}^{\prime} h_{j}\right)\left(\sum_{k=1}^{r} a_{k}^{\prime \prime} l_{k}\right) \\
& +\left(\sum_{i=1}^{n} b_{i} g_{i}\right)\left(\sum_{j=1}^{m} d_{j}^{\prime} h_{j}\right)\left(\sum_{k=1}^{r} a_{k}^{\prime \prime} l_{k}\right)+\left(\sum_{i=1}^{n} c_{i} g_{i}\right)\left(\sum_{j=1}^{m} a_{j}^{\prime} h_{j}\right)\left(\sum_{k=1}^{r} a_{k}^{\prime \prime} l_{k}\right) \\
& =0, \\
& \left(\sum_{i=1}^{n} a_{i} g_{i}\right)\left(\sum_{j=1}^{m} a_{j}^{\prime} h_{j}\right)\left(\sum_{k=1}^{r} d_{k}^{\prime \prime} l_{k}\right)+\left(\sum_{i=1}^{n} a_{i} g_{i}\right)\left(\sum_{j=1}^{m} d_{j}^{\prime} h_{j}\right)\left(\sum_{k=1}^{r} a_{k}^{\prime \prime} l_{k}\right) \\
& +\left(\sum_{i=1}^{n} d_{i} g_{i}\right)\left(\sum_{j=1}^{m} a_{j}^{\prime} h_{j}\right)\left(\sum_{k=1}^{r} a_{k}^{\prime \prime} l_{k}\right)=0 .
\end{aligned}
$$

Since $R$ is 3 - $M$-Armendariz we have $a_{i} a_{j}^{\prime} a_{k}^{\prime \prime}=0$ for all $i, j$ and $k$. Thus, $a_{k}^{\prime \prime} a_{i} a_{j}^{\prime}=a_{j}^{\prime} a_{k}^{\prime \prime} a_{i}=0$, since $R$ satisfies condition $(P)$. Hence

$$
\left(\sum_{k=1}^{r} a_{k}^{\prime \prime} l_{k}\right)\left(\sum_{i=1}^{n} a_{i} g_{i}\right)\left(\sum_{j=1}^{m} a_{j}^{\prime} h_{j}\right)=\left(\sum_{j=1}^{m} a_{j}^{\prime} h_{j}\right)\left(\sum_{k=1}^{r} a_{k}^{\prime \prime} l_{k}\right)\left(\sum_{i=1}^{n} a_{i} g_{i}\right)=0
$$

for all $i, j$ and $k$. If we multiply the equation (2) on left side by $\left(\sum_{k=1}^{r} a_{k}^{\prime \prime} l_{k}\right)$ then

$$
\begin{aligned}
& \left(\sum_{k=1}^{r} a_{k}^{\prime \prime} l_{k}\right)\left(\sum_{i=1}^{n} a_{i} g_{i}\right)\left(\sum_{j=1}^{m} b_{j}^{\prime} h_{j}\right)\left(\sum_{k=1}^{r} a_{k}^{\prime \prime} l_{k}\right) \\
& +\left(\sum_{k=1}^{r} a_{k}^{\prime \prime} l_{k}\right)\left(\sum_{i=1}^{n} b_{i} g_{i}\right)\left(\sum_{j=1}^{m} a_{j}^{\prime} h_{j}\right)\left(\sum_{k=1}^{r} a_{k}^{\prime \prime} l_{k}\right)=0 .
\end{aligned}
$$

Multiplying (5) on left side by $\left(\sum_{j=1}^{m} a_{j}^{\prime} h_{j}\right)$ then we have

$$
\left(\sum_{j=1}^{m} a_{j}^{\prime} h_{j}\right)\left(\sum_{k=1}^{r} a_{k}^{\prime \prime} l_{k}\right)\left(\sum_{i=1}^{n} b_{i} g_{i}\right)\left(\sum_{j=1}^{m} a_{j}^{\prime} h_{j}\right)\left(\sum_{k=1}^{r} a_{k}^{\prime \prime} l_{k}\right)=0 .
$$

Since $R$ is $3-M$-Armendariz ring and satisfies condition $(P)$, by Proposition 2.11 it follows that $a_{j}^{\prime} a_{k}^{\prime \prime} b_{i} a_{j}^{\prime} a_{k}^{\prime \prime}=0$. Thus, $b_{i} a_{j}^{\prime} a_{k}^{\prime \prime}=0$ for all $i, j$ and $k$. Now (5) becomes

$$
\left(\sum_{k=1}^{r} a_{k}^{\prime \prime} l_{k}\right)\left(\sum_{i=1}^{n} a_{i} g_{i}\right)\left(\sum_{j=1}^{m} b_{j}^{\prime} h_{j}\right)\left(\sum_{k=1}^{r} a_{k}^{\prime \prime} l_{k}\right)=0 .
$$

Thus, $a_{i} b_{j}^{\prime} a_{k}^{\prime \prime}=0$ for all $i, j$ and $k$, since $R$ is 3 - $M$-Armendariz and satisfies condition $(P)$. Hence from $(2)$ it follows

$$
\left(\sum_{i=1}^{n} a_{i} g_{i}\right)\left(\sum_{j=1}^{m} a_{j}^{\prime} h_{j}\right)\left(\sum_{k=1}^{r} b_{k}^{\prime \prime} l_{k}\right)=0 .
$$


Thus, $a_{i} a_{j}^{\prime} b_{k}^{\prime \prime}=0$ for all $i, j$ and $k$. Now multiplying (4) on left side by $\left(\sum_{k=1}^{r} a_{k}^{\prime \prime} l_{k}\right)$ yields

$$
\begin{aligned}
& \left(\sum_{k=1}^{r} a_{k}^{\prime \prime} l_{k}\right)\left(\sum_{j=1}^{m} a_{i} g_{i}\right)\left(\sum_{j=1}^{m} d_{j}^{\prime} h_{j}\right)\left(\sum_{k=1}^{r} a_{k}^{\prime \prime} l_{k}\right) \\
& +\left(\sum_{k=1}^{r} a_{k}^{\prime \prime} l_{k}\right)\left(\sum_{i=1}^{n} d_{i} g_{i}\right)\left(\sum_{j=1}^{m} a_{j}^{\prime} h_{j}\right)\left(\sum_{k=1}^{r} a_{k}^{\prime \prime} l_{k}\right)=0 .
\end{aligned}
$$

Multiplying (7) on left side by $\left(\sum_{j=1}^{m} a_{j}^{\prime} h_{j}\right)$ then we have

$$
\left(\sum_{j=1}^{m} a_{j}^{\prime} h_{j}\right)\left(\sum_{k=1}^{r} a_{k}^{\prime \prime} l_{k}\right)\left(\sum_{i=1}^{n} d_{i} g_{i}\right)\left(\sum_{j=1}^{m} a_{j}^{\prime} h_{j}\right)\left(\sum_{k=1}^{r} a_{k}^{\prime \prime} l_{k}\right)=0 .
$$

Thus, $d_{i} a_{j}^{\prime} a_{k}^{\prime \prime}=0$ for all $i, j$ and $k$, since $R$ satisfies condition $(P)$. Similarly, we have

$$
\left(\sum_{k=1}^{r} a_{k}^{\prime \prime} l_{k}\right)\left(\sum_{j=1}^{m} a_{i} g_{i}\right)\left(\sum_{j=1}^{m} d_{j}^{\prime} h_{j}\right)\left(\sum_{k=1}^{r} a_{k}^{\prime \prime} l_{k}\right)=0 .
$$

Thus, $a_{i} d_{j}^{\prime} a_{k}^{\prime \prime}=0$ for all $i, j$ and $k$. Now from (4) it follows

$$
\left(\sum_{i=1}^{n} a_{i} g_{i}\right)\left(\sum_{j=1}^{m} a_{j}^{\prime} h_{j}\right)\left(\sum_{k=1}^{r} d_{k}^{\prime \prime} l_{k}\right)=0 .
$$

Thus, $a_{i} a_{j}^{\prime} d_{k}^{\prime \prime}=0$ for all $i, j$ and $k$. Now we multiply (3) on left side by $\left(\sum_{k=1}^{r} a_{k}^{\prime \prime} l_{k}\right)$ then

$$
\begin{aligned}
& \left(\sum_{k=1}^{r} a_{k}^{\prime \prime} l_{k}\right)\left(\sum_{i=1}^{n} a_{i} g_{i}\right)\left(\sum_{j=1}^{m} c_{j}^{\prime} h_{j}\right)\left(\sum_{k=1}^{r} a_{k}^{\prime \prime} l_{k}\right) \\
& +\left(\sum_{k=1}^{r} a_{k}^{\prime \prime} l_{k}\right)\left(\sum_{i=1}^{n} b_{i} g_{i}\right)\left(\sum_{j=1}^{m} d_{j}^{\prime} h_{j}\right)\left(\sum_{k=1}^{r} a_{k}^{\prime \prime} l_{k}\right) \\
& +\left(\sum_{k=1}^{r} a_{k}^{\prime \prime} l_{k}\right)\left(\sum_{i=1}^{n} c_{i} g_{i}\right)\left(\sum_{j=1}^{m} a_{j}^{\prime} h_{j}\right)\left(\sum_{k=1}^{r} a_{k}^{\prime \prime} l_{k}\right)=0 .
\end{aligned}
$$

Multiplying (8) on left side by $\left(\sum_{j=1}^{m} a_{j}^{\prime} h_{j}\right)$ yileds

$$
\left(\sum_{j=1}^{m} a_{j}^{\prime} h_{j}\right)\left(\sum_{k=1}^{r} a_{k}^{\prime \prime} l_{k}\right)\left(\sum_{i=1}^{n} c_{i} g_{i}\right)\left(\sum_{j=1}^{m} a_{j}^{\prime} h_{j}\right)\left(\sum_{k=1}^{r} a_{k}^{\prime \prime} l_{k}\right)=0 .
$$

Thus, $c_{i} a_{j}^{\prime} a_{k}^{\prime \prime}=0$ for all $i, j$ and $k$. Thus (8) becomes

$$
\begin{aligned}
& \left(\sum_{k=1}^{r} a_{k}^{\prime \prime} l_{k}\right)\left(\sum_{i=1}^{n} a_{i} g_{i}\right)\left(\sum_{j=1}^{m} c_{j}^{\prime} h_{j}\right)\left(\sum_{k=1}^{r} a_{k}^{\prime \prime} l_{k}\right) \\
& +\left(\sum_{k=1}^{r} a_{k}^{\prime \prime} l_{k}\right)\left(\sum_{i=1}^{n} b_{i} g_{i}\right)\left(\sum_{j=1}^{m} d_{j}^{\prime} h_{j}\right)\left(\sum_{k=1}^{r} a_{k}^{\prime \prime} l_{k}\right)=0 .
\end{aligned}
$$

Multiplying (9) on left side by $\left(\sum_{j=1}^{m} d_{j}^{\prime} h_{j}\right)$ yields

$$
\left(\sum_{j=1}^{m} d_{j}^{\prime} h_{j}\right)\left(\sum_{k=1}^{r} a_{k}^{\prime \prime} l_{k}\right)\left(\sum_{i=1}^{n} b_{i} g_{i}\right)\left(\sum_{j=1}^{m} d_{j}^{\prime} h_{j}\right)\left(\sum_{k=1}^{r} a_{k}^{\prime \prime} l_{k}\right)=0
$$


Thus, we have $b_{i} d_{j}^{\prime} a_{k}^{\prime \prime}=0$ for all $i, j$ and $k$. Now from (8) it follows

$$
\left(\sum_{k=1}^{r} a_{k}^{\prime \prime} l_{k}\right)\left(\sum_{i=1}^{n} a_{i} g_{i}\right)\left(\sum_{j=1}^{m} c_{j}^{\prime} h_{j}\right)\left(\sum_{k=1}^{r} a_{k}^{\prime \prime} l_{k}\right)=0 .
$$

Thus, $a_{i} c_{j}^{\prime} a_{k}^{\prime \prime}=0$ for all $i, j$ and $k$. Now (3) becomes

$$
\begin{aligned}
& \left(\sum_{i=1}^{n} a_{i} g_{i}\right)\left(\sum_{j=1}^{m} a_{j}^{\prime} h_{j}\right)\left(\sum_{k=1}^{r} c_{k}^{\prime \prime} l_{k}\right)+\left(\sum_{i=1}^{n} a_{i} g_{i}\right)\left(\sum_{j=1}^{m} b_{j}^{\prime} h_{j}\right)\left(\sum_{k=1}^{r} d_{k}^{\prime \prime} l_{k}\right) \\
& +\left(\sum_{i=1}^{n} b_{i} g_{i}\right)\left(\sum_{j=1}^{m} a_{j}^{\prime} h_{j}\right)\left(\sum_{k=1}^{r} d_{k}^{\prime \prime} l_{k}\right)=0 .
\end{aligned}
$$

Multiplying (10) on left side by $\left(\sum_{k=1}^{r} d_{k}^{\prime \prime} l_{k}\right)$ yields

$$
\begin{aligned}
& \left(\sum_{k=1}^{r} d_{k}^{\prime \prime} l_{k}\right)\left(\sum_{i=1}^{n} a_{i} g_{i}\right)\left(\sum_{j=1}^{m} b_{j}^{\prime} h_{j}\right)\left(\sum_{k=1}^{r} d_{k}^{\prime \prime} l_{k}\right) \\
& +\left(\sum_{k=1}^{r} d_{k}^{\prime \prime} l_{k}\right)\left(\sum_{i=1}^{n} b_{i} g_{i}\right)\left(\sum_{j=1}^{m} a_{j}^{\prime} h_{j}\right)\left(\sum_{k=1}^{r} d_{k}^{\prime \prime} l_{k}\right)=0 .
\end{aligned}
$$

Multiplying (11) on left side by $\left(\sum_{j=1}^{m} a_{j}^{\prime} h_{j}\right)$ then

$$
\left(\sum_{j=1}^{m} a_{j}^{\prime} h_{j}\right)\left(\sum_{k=1}^{r} d_{k}^{\prime \prime} l_{k}\right)\left(\sum_{i=1}^{n} b_{i} g_{i}\right)\left(\sum_{j=1}^{m} a_{j}^{\prime} h_{j}\right)\left(\sum_{k=1}^{r} d_{k}^{\prime \prime} l_{k}\right)=0 .
$$

Thus, $b_{i} a_{j}^{\prime} d_{k}^{\prime \prime}=0$ for all $i, j$ and $k$. Thus (11) becomes

$$
\left(\sum_{k=1}^{r} d_{k}^{\prime \prime} l_{k}\right)\left(\sum_{i=1}^{n} a_{i} g_{i}\right)\left(\sum_{j=1}^{m} b_{j}^{\prime} h_{j}\right)\left(\sum_{k=1}^{r} d_{k}^{\prime \prime} l_{k}\right)=0 .
$$

Thus, we have $a_{i} b_{j}^{\prime} d_{k}^{\prime \prime}=0$ for all $i, j$ and $k$. Now from (10) it follows

$$
\left(\sum_{i=1}^{n} a_{i} g_{i}\right)\left(\sum_{j=1}^{m} a_{j}^{\prime} h_{j}\right)\left(\sum_{k=1}^{r} c_{k}^{\prime \prime} l_{k}\right)=0 .
$$

Thus, $a_{i} a_{j}^{\prime} c_{k}^{\prime \prime}=0$ for all $i, j$ and $k$. Now it is easy to see that $A_{i} B_{j} C_{k}=0$ for all $i, j$ and $k$.

Then $S_{3}(R)$ is 3 - $M$-Armendariz.

Remark 2.13. Let $R$ be a ring and let

$$
S_{n}(R)=\left\{\left(\begin{array}{ccccc}
a & a_{12} & a_{13} & \cdots & a_{1 n} \\
0 & a & a_{23} & \cdots & a_{2 n} \\
0 & 0 & a & \cdots & a_{3 n} \\
\cdots & \cdots & \cdots & \ddots & \cdots \\
0 & 0 & 0 & 0 & a
\end{array}\right) a, a_{i j} \in R\right\} .
$$


Where $n$ is a positive integer. Then Theorem 2.12 suggests that $S_{n}(R)$ may be also 3-M-Armendariz for $n \geq 4$ if $R$ is 3-M-Armendariz and satisfies condition $(P)$. But the examples appeared in Kim and Lee [9, Example 3], and Hong et al. [2, Example 18], eliminate the possibility.

Example 2.14. Let $M$ be a monoid with $|M| \geq 2$ and $R$ a ring with identity. Take $e \neq g \in M$. Let $\alpha=E_{13} e+\left(E_{13}-E_{12}\right) g, \beta=E_{24} e+\left(E_{24}+E_{34}\right) g, \gamma=$ $I_{4} e$, be in $S_{n}(R)[M]$, where $E_{i j}$ 's are the matrix units in $S_{n}(R)(n \geq 4)$. Then $\alpha \beta \gamma=0$, but $E_{13}\left(E_{24}+E_{34}\right) I_{4} \neq 0$. Thus, $S_{n}(R)$ is not 3-M-Armendariz $(n \geq 4)$.

Corollary 2.15. Let $M$ be a monoid and $R 3$ - $M$-Armendariz ring. If $R$ satisfies condition $(P)$, then the trivial extension $T(R, R)$ is $3-M$-Armendariz.

Proof. Note that $T(R, R)$ is isomorphic to the ring

$$
\left\{\left(\begin{array}{lll}
a & b & 0 \\
0 & a & 0 \\
0 & 0 & a
\end{array}\right)^{\mid a, b \in R}\right\} .
$$

Now the result follows from Theorem 2.12 and from the fact that every subring of a 3 - $M$-Armendariz ring is $3-M$-Armendariz.

Recall that an element $u$ of a ring $R$ is right regular if $u r=0$ implies $r=0$ for $r \in R$. Similarly, left regular elements can be defined. An element is regular if it is both left and right regular (and hence not a zero divisor).

Proposition 2.16. Let $R$ be a ring and $\Delta$ be a multiplicative monoid in $R$ consisting of central regular elements. Then $R$ is $3-M$-Armendariz if and only if $\Delta^{-1} R$ is also $3-M$-Armendariz.

Proof. Let $R$ be a 3 - $M$-Armendariz ring, and $S=\Delta^{-1} R$. Put $\alpha \beta \gamma=0$, where $\alpha=\sum_{i=1}^{n} a_{i} g_{i}, \beta=\sum_{j=1}^{m} b_{j} h_{j}$ and $\gamma=\sum_{k=1}^{r} c_{k} l_{k} \in S[M]$. We may assume that $a_{i}=\varepsilon_{i} u^{-1}, b_{j}=\eta_{j} v^{-1}$ and $c_{k}=\mu_{k} w^{-1}$ with $\varepsilon_{i}, \eta_{j}, \mu_{k}$ are in $R$ for all $i, j$ and $k$, and $u, v, w \in \Delta$. Then we have

$$
\begin{gathered}
0=\alpha \beta \gamma=\sum_{i=1}^{n} \sum_{j=1}^{m} \sum_{k=1}^{r} a_{i} b_{j} c_{k} g_{i} h_{j} l_{k} \\
=\sum_{i=1}^{n} \sum_{j=1}^{m} \sum_{k=1}^{r} \varepsilon_{i} \eta_{j} \mu_{k} u^{-1} v^{-1} w^{-1} g_{i} h_{j} l_{k}
\end{gathered}
$$




$$
=\left(\sum_{i=1}^{n} \sum_{j=1}^{m} \sum_{k=1}^{r} \varepsilon_{i} \eta_{j} \mu_{k} g_{i} h_{j} l_{k}\right)(u v w)^{-1} .
$$

Hence

$$
\sum_{i=1}^{n} \sum_{j=1}^{m} \sum_{k=1}^{r} \varepsilon_{i} \eta_{j} \mu_{k} g_{i} h_{j} l_{k}=0
$$

in $R[M]$. Since $R$ is $3-M$-Armendariz, $\varepsilon_{i} \eta_{j} \mu_{k}=0$, for all $i, j$ and $k$ and so

$$
a_{i} b_{j} c_{k}=\varepsilon_{i} u^{-1} \eta_{j} v^{-1} \mu_{k} w^{-1}=\varepsilon_{i} \eta_{j} \mu_{k}(u v w)^{-1}=0,
$$

for all $i, j, k$. Thus, $S$ is 3 - $M$-Armendariz. The converse follows from Proposition 2.3 .

Proposition 2.17. Let $M$ be a monoid, $R_{1}$ and $R_{2}$ rings. Then the following conditions are equivalent:

1. $R_{1} \times R_{2}$ is $3-M-$ Armendariz;

2. $R_{1}$ and $R_{2}$ are $3-M-A r m e n d a r i z$.

Proof. $(2) \Rightarrow(1)$. Let $R_{1}$ and $R_{2}$ are 3-M-Armendariz. Suppose that $\alpha=$ $a_{1} g_{1}+\cdots+a_{n} g_{n}, \beta=b_{1} h_{1}+\cdots+b_{m} h_{m}$ and $\gamma=c_{1} l_{1}+\cdots+c_{r} l_{r} \in\left(R_{1} \times R_{2}\right)[M]$, such that $\alpha \beta \gamma=0$, where $a_{i}, b_{j}, c_{k}$ are in $\left(R_{1} \times R_{2}\right)$, and $g_{i}, h_{j}, l_{k}$ are in $M$ for all $i, j$ and $k$. Then

$$
\sum_{g \in M}\left(\sum_{g_{i} h_{j} l_{k}=g}\left(a_{i} b_{j} c_{k}\right)\right) g=0
$$

Thus,

$$
\sum_{g_{i} h_{j} l_{k}=g} a_{i} b_{j} c_{k}=0, \forall g \in M .
$$

Set $a_{i}=\left(a_{i}^{1}, a_{i}^{2}\right), b_{j}=\left(b_{j}^{1}, b_{j}^{2}\right), c_{k}=\left(c_{k}^{1}, c_{k}^{2}\right)$, where $a_{i}^{1}, b_{j}^{1}, c_{k}^{1} \in R_{1}$ and $a_{i}^{2}, b_{j}^{2}, c_{k}^{2} \in$ $R_{2}$ for all $i, j$ and $k$. Then

$$
\sum_{g_{i} h_{j} l_{k}=g} a_{i}^{1} b_{j}^{1} c_{k}^{1}=0, \sum_{g_{i} h_{j} l_{k}=g} a_{i}^{2} b_{j}^{2} c_{k}^{2}=0, \forall g \in M .
$$

Thus,

$$
\left(\sum_{i} a_{i}^{1} g_{i}\right)\left(\sum_{j} b_{j}^{1} h_{j}\right)\left(\sum_{k} c_{k}^{1} l_{k}\right)=\sum_{g \in M}\left(\sum_{g_{i} h_{j} l_{k}=g} a_{i}^{1} b_{j}^{1} c_{k}^{1}\right) g=0 .
$$

Similarly, $\left(\sum_{i} a_{i}^{2} g_{i}\right)\left(\sum_{j} b_{j}^{2} h_{j}\right)\left(\sum_{k} c_{k}^{2} l_{k}\right)=0$. Since $R_{1}$ and $R_{2}$ are 3 - $M$-Armendariz, we have $a_{i}^{1} b_{j}^{1} c_{k}^{1}=0$ and $a_{i}^{2} b_{j}^{2} c_{k}^{2}=0$ for all $i, j$ and $k$. Thus, $a_{i} b_{j} c_{k}=0$ for all $i, j$ and $k$. This shows that $R_{1} \times R_{2}$ is 3 - $M$-Armendariz. 
$(1) \Rightarrow(2)$. This is clear since $R_{1} \cong R_{1} \times\{0\}$ and $R_{2} \cong\{0\} \times R_{2}$.

Corollary 2.18. Let $M$ be a monoid and $R_{i}, i \in I$, be rings. Then the following statements are equivalent:

1. $\prod_{i \in I} R_{i}$ is 3 -M-Armendariz;

2. $\bigoplus_{i \in I} R_{i}$ is $3-M$-Armendariz;

3. $R_{i}$ is 3-M-Armendariz for each $i \in I$.

Proof. By the same method as above, we have $(1) \Leftrightarrow(3)$ and $(2) \Leftrightarrow(3)$.

\section{Monoid Rings}

Anderson and Camillo [3, Theorem 2], have shown that a ring $R$ is Armendariz if and only if $R[x]$ is Armendariz, and Zhongkui [7, proposition 2.1], have shown that if $R$ is a reduced and $M$-Armendariz ring, then $R[M]$ is $N$-Armendariz, where $M$ is a monoid and $N$ a u.p.-monoid. For 3-M-Armendariz, we have the following results.

Proposition 3.1. Let $M$ be a monoid and $N$ a u.p.-monoid. If $R$ satisfies condition $(P)$ and is 3-M-Armendariz, then $R[M]$ is $3-N$-Armendariz.

Proof. Suppose that $\alpha=a_{1} g_{1}+\cdots+a_{n} g_{n}, \beta=b_{1} h_{1}+\cdots+b_{m} h_{m}$ and $\gamma=c_{1} l_{1}+\cdots+c_{k} l_{k} \in R[M]$, such that $(\alpha \beta \gamma)^{2}=0$. Then $\left(a_{i} b_{j} c_{k}\right)^{2}=0$, for all $i, j$ and $k$, since $R$ is 3-M-Armendariz. Thus, $\left(a_{i} b_{j} c_{k}\right)=0$, for all $i, j$ and $k$, since $R$ satisfies condition $(P)$. Hence $\alpha \beta \gamma=0$. This shows that $R[M]$ satisfies condition $(P)$. Now the result follows from Theorem 2.6.

Proposition 3.2. Let $M$ be a monoid and $N$ a u.p.-monoid. If $R$ satisfies condition $(P)$, and is $3-M$-Armendariz, then $R[N]$ is $3-M$-Armendariz.

Proof. There exists an isomorphism of rings $R[N][M] \cong R[M][N]$ defined by

$$
\sum_{p}\left(\sum_{i} a_{i p} n_{i}\right) m_{p} \rightarrow \sum_{i}\left(\sum_{p} a_{i p} m_{p}\right) n_{i} .
$$

Now suppose that $\alpha_{i}, \beta_{j}, \gamma_{k} \in R[N]$ are such that $\left(\sum_{i} \alpha_{i} g_{i}\right)\left(\sum_{j} \beta_{j} h_{j}\right)\left(\sum_{k} \gamma_{k} l_{k}\right)=$ 0 , where $g_{i}, h_{j}, l_{k} \in M$. We will show that $\alpha_{i} \beta_{j} \gamma_{k}=0$, for all $i, j$ and $k$. Assume 
that $\alpha_{i}=\sum_{p} a_{i p} n_{p}, \beta_{j}=\sum_{q} b_{j q} n_{q}^{\prime}$ and $\gamma_{k}=\sum_{s} c_{k s} n_{s}^{\prime \prime}$, where $n_{p}, n_{q}^{\prime}, n_{s}^{\prime \prime} \in N$ for all $p, q$ and $s$. Then

$$
\left(\sum_{i}\left(\sum_{p} a_{i p} n_{p}\right) g_{i}\right)\left(\sum_{j}\left(\sum_{q} b_{j q} n_{q}^{\prime}\right) h_{j}\right)\left(\sum_{k}\left(\sum_{s} c_{k s} n_{s}^{\prime \prime}\right) l_{k}\right)=0 .
$$

Thus, in $R[M][N]$ we have

$$
\left(\sum_{p}\left(\sum_{i} a_{i p} g_{i}\right) n_{p}\right)\left(\sum_{q}\left(\sum_{j} b_{j q} h_{j}\right) n_{q}^{\prime}\right)\left(\sum_{s}\left(\sum_{k} c_{k s} l_{k}\right) n_{s}^{\prime \prime}\right)=0 .
$$

By Proposition 3.1, $R[M]$ is $3-N$-Armendariz. Thus

$$
\left(\sum_{i} a_{i p} g_{i}\right)\left(\sum_{j} b_{j q} h_{j}\right)\left(\sum_{k} c_{k s} l_{k}\right)=0
$$

for all $p, q$ and $s$. So $a_{i p} b_{j q} c_{k s}=0$ for all $i, j, k, p, q, s$, since $R$ is 3 - $M$-Armendariz. Hence

$$
\alpha_{i} \beta_{j} \gamma_{k}=\left(\sum_{p} a_{i p} n_{p}\right)\left(\sum_{q} b_{j q} n_{q}^{\prime}\right)\left(\sum_{s} c_{k s} n_{s}^{\prime \prime}\right)=0,
$$

for all $p, q$ and $s$.

Theorem 3.3. Let $M$ be a monoid and $N$ a u.p.-monoid. If $R$ satisfies condition $(P)$, and is $3-M$-Armendariz, then $R$ is $3-(M \times N)$-Armendariz.

Proof. Suppose that $\sum_{i=1}^{s} a_{i}\left(m_{i}, n_{i}\right)$ is in $R[M \times N]$. Without loss of generality, we assume that $\left\{n_{1}, n_{2}, \cdots, n_{s}\right\}=\left\{n_{1}, n_{2}, \cdots, n_{t}\right\}$ with $n_{i} \neq n_{j}$ when $1 \leq i \neq j \leq t$. For any $1 \leq p \leq t$, denote $A_{p}=\left\{i \mid 1 \leq i \leq s, n_{i}=n_{p}\right\}$. Then $\sum_{p=1}^{t} \sum_{i \in A_{p}}\left(a_{i} m_{i}\right) n_{p} \in R[M][N]$. Note that $m_{i} \neq m_{i^{\prime}}$ for any $i, i^{\prime} \in A_{p}$ with $i \neq i^{\prime}$. Now it is easy to see that there exists an isomorphism of rings $R[M \times N] \rightarrow R[M][N]$ define by

$$
\sum_{i=1}^{s} a_{i}\left(m_{i}, n_{i}\right) \rightarrow \sum_{p=1}^{t} \sum_{i \in A_{p}}\left(a_{i} m_{i}\right) n_{p}
$$

Suppose that

$$
\left(\sum_{i=1}^{s} a_{i}\left(m_{i}, n_{i}\right)\right)\left(\sum_{j=1}^{s^{\prime}} b_{j}\left(m_{j}^{\prime}, n_{j}^{\prime}\right)\right)\left(\sum_{k=1}^{s^{\prime \prime}} c_{k}\left(m_{k}^{\prime \prime}, n_{k}^{\prime \prime}\right)\right)=0
$$


in $R[M \times N]$. Then from the above isomorphism it follows that

$$
\left(\sum_{p=1}^{t}\left(\sum_{i \in A_{p}} a_{i} m_{i}\right) n_{p}\right)\left(\sum_{q=1}^{t^{\prime}}\left(\sum_{j \in B_{q}} b_{j} m_{j}^{\prime}\right) n_{q}^{\prime}\right)\left(\sum_{r=1}^{t^{\prime \prime}}\left(\sum_{k \in C_{r}} c_{k} m_{k}^{\prime \prime}\right) n_{r}^{\prime \prime}\right)=0 .
$$

By Proposition $3.1 R[M]$ is 3-N-Armendariz. Thus we have

$$
\left(\sum_{i \in A_{p}} a_{i} m_{i}\right)\left(\sum_{j \in B_{q}} b_{j} m_{j}^{\prime}\right)\left(\sum_{k \in C_{r}} c_{k} m_{k}^{\prime \prime}\right)=0
$$

for any $p, q$ and $r$. Hence $a_{i} b_{j} c_{k}=0$, for any $i \in A_{p}, j \in B_{q}$ and any $k \in C_{r}$, since $R$ is 3 - $M$-Armendariz. Thus, $a_{i} b_{j} c_{k}=0$ for all $i, j, k$, such that $1 \leq i \leq$ $s, 1 \leq j \leq s^{\prime}, 1 \leq k \leq s^{\prime \prime}$. This means that $R$ is 3 - $(M \times N)$-Armendariz.

Let $M_{i}, i \in I$, be monoids. Denote $\coprod_{i \in I} M_{i}=\left\{\left(g_{i}\right)_{i \in I} \mid\right.$ there exist only finite $i$ 's such that $g_{i} \neq e_{i}$, the identity of $\left.M_{i}\right\}$. Then $\coprod_{i \in I} M_{i}$ is a monoid with the operation $\left(g_{i}\right)_{i \in I}\left(g_{i}^{\prime}\right)_{i \in I}=\left(g_{i} g_{i}^{\prime}\right)_{i \in I}$.

Corollary 3.4. Let $M_{i}, i \in I$ be u.p.-monoids and a ring $R$ satisfies condition $(P)$. If $R$ is $3-M_{i_{0}}-$ Armendariz for some $i_{0} \in I$, then $R$ is $3-\coprod_{i \in I} M_{i^{-}}$ Armendariz.

Proof. Let $\alpha=\sum_{i} a_{i} g_{i}, \beta=\sum_{j} b_{j} h_{j}, \gamma=\sum_{k} c_{k} l_{k} \in R\left[\amalg_{i \in I} M_{i}\right]$ such that $\alpha \beta \gamma=0$. Then $\alpha, \beta, \gamma \in R\left[M_{1} \times M_{2} \times \cdots \times M_{n}\right]$, for some finite subset $\left\{M_{1}, M_{2}, \cdots, M_{n}\right\} \subseteq\left\{M_{i} \mid i \in I\right\}$. Thus $\alpha, \beta, \gamma \in R\left[M_{i_{0}} \times M_{1} \times M_{2} \times \cdots \times M_{n}\right]$. The ring $R$, by Theorem 3.3 and by induction, is $3-\left(M_{i_{0}} \times M_{1} \times M_{2} \times \cdots \times\right.$ $M_{n}$ )-Armendariz, so $a_{i} b_{j} c_{k}=0$ for all $i, j$ and $k$. Hence $R$ is $3-\coprod_{i \in I} M_{i^{-}}$ Armendariz.

Corollary 3.5. Let $M$ be a monoid and a ring $R$ satisfies condition $(P)$. If $R$ is 3 - $M$-Armendariz, then $R[x]$ and $R\left[x, x^{-1}\right]$ are 3 -M-Armendariz.

Proof. Note that $R[x] \cong R[\mathbb{N} \cup\{0\}]$ and $R\left[x, x^{-1}\right] \cong R[\mathbb{Z}]$.

\section{Acknowledgments}

We would like to thank the referees for a very careful reading of the paper and their valuable comments.

This paper was partially supported by National Natural Science Foundation of China (No. 11261050). 


\section{References}

[1] C. Huh, Y. Lee, A. Smoktunowicz, Armendariz rings and semicommutative rings, Comm. Algebra, 30, No. 2 (2002), 751-761.

[2] C.Y. Hong, N.K. Kim, T.K. Kwak, On Skew Armendariz rings, Comm. Algebra, 31, No. 1 (2003), 103-122.

[3] D.D. Anderson, V. Camillo, Armendariz rings and Gaussian rings, Comm. Algebra, 26, No. 7 (1998), 2265-2272.

[4] D.S. Passman, The Algebraic Structure of Group Rings, John Wiley, New York (1977).

[5] E.P. Armendariz, A note on extensions of Baer and p.p.-rings, J. Austral. Math. Soc., 18 (1974), 470-473.

[6] G.F. Birkenmeier, J.K. Park, Triangular matrix representations of ring extensions, J. Algebra, 265 (2003), 457-477.

[7] L. Zhongkui, Armendariz rings relative to a monoid, Comm. Algebra, 33, No. 3 (2005), 649-661.

[8] M.B. Rege, S. Chhawchharia, Armendariz rings, Proc. Japan Acad. Ser. A Math. Sci., 73 (1997), 14-17.

[9] N.K. Kim, Y. Lee, Armendariz rings and reduced rings, J. Algebra, 223 (2000), 477-488.

[10] P. Ribenboim, Noetherian rings of generalized power series, J. Pure Appl. Algebra, 79 (1992), 293-312.

[11] T.K. Lee, T.L. Wong, On Armendariz rings, Houston J. Math., 29, No. 3 (2003), 583-593.

[12] T.K. Lee, Y.Q. Zhou, Armendariz and reduced rings, J. Comm. Algebra, 32, No. 6 (2004), 2287-2299.

[13] Wu Hui-Feng, Extensions of Reduced Rings, J. Hangzhou Normal Uni., 10, No. 5 (2011), 407-410.

[14] Y. Suiyi, On the Extension of Armendariz Rings, Lanzhou University (2008). 